\title{
The Applications of Meshfree Particle Methods at the Nanoscale
}

\author{
Weixuan Yang and Shaoping Xiao \\ Department of Mechanical and Industrial Engineering and Center for Computer-Aided \\ Design, The University of Iowa, 3131 Seamans Center, Iowa City, IA, 52241-1527, USA \\ \{weixuan-yang, shaoping-xiao\} @uiowa.edu
}

\begin{abstract}
Since meshfree particle methods are beneficial in simulating the problems involving extremely large deformations, fractures, etc., these methods become attractive options in multiscale modeling, especially when approaching a large number of atoms. In this paper, we propose preliminary research on applying meshfree particle methods to solve nanoscale problems. A quasicontinuum technique, i.e. the Cauchy-Born rule, is implemented into the meshfree particle methods so continuum approaches for large deformation problems or fracture problems at the nanoscale can be performed. Furthermore, the meshfree particle methods can be coupled with molecular dynamics via the bridging domain coupling technique. The examples show that the meshfree particle methods can benefit either hierarchical or concurrent multiscale modeling at the nanoscale.
\end{abstract}

\section{Introduction}

With the development of nanotechnology, numerical simulation plays an important role in nanoscale material and device design. To develop a potential numerical method, which can efficiently model micro/nano systems, has been one of the forefront research topics of computational nanotechnology.

Among a variety of numerical simulation techniques, molecular dynamics (MD) has become a powerful tool to elucidate complex physical phenomena [1-2]. Up to billions of atoms can be simulated by MD when studying the crack propagation [2] at the atomistic level with parallel computing techniques. However, most MD simulations are still restricted on both small length and short time scales. Therefore, multiscale methods have been of more and more interest to simulate large nanoscale systems. The recently developed multiscale methods can be divided into two classes: hierarchical multiscale methods [3] and concurrent multiscale methods [4-7]. In hierarchical multiscale modeling, the continuum approximation is based on the properties of a subscale model, such as a MD model. The intrinsic properties of materials are sought at the atomic level and embedded in the continuum model according to the quasicontinuum technique, which is also called the Cauchy-Born rule [8-9]. The Cauchy-Born rule states that the deformation is locally homogeneous.

Concurrent multiscale methods use an appropriate model to solve each length scale simultaneously. Recently, some concurrent multiscale techniques [4-6], particularly coupling methods between the continuum model and the molecular model, have been 
developed. One of the key issues the concurrent multiscale methods must overcome is the occurrence of spurious numerical phenomena, such as non-physical reflections on the interfaces between the molecular and continuum models. Most researchers use the Langevin equation [6] or other filtering processes to eliminate spurious reflections. Xiao and Belytschko developed a bridging domain coupling method [7], which can eliminate the spurious wave reflection automatically.

Mostly, finite element methods are used in the hierarchical or concurrent multiscale methods with the implementation of the quasicontinuum technique. It is known that the meshfree particle methods [10] are more attractive for a variety of problems with moving boundaries, discontinuities, and extremely large deformations. Therefore, the incorporation of the meshfree particle methods and the quasicontinuum technique will have much potential to solve the above problems at the nanoscale. Belytschko and Xiao [11] found that the meshfree particle methods with Lagrangian kernels are more stable than those with Eulerian kernels. In this paper, only the meshfree particle methods with Lagrangian kernels are considered. With the implementation of the quasicontinuum method, the meshfree particle methods can be used to simulate large nano systems. Furthermore, based on the idea of the bridging domain coupling method [7], the meshfree particle methods can be coupled with molecular dynamics to accomplish a multiscale modeling for large nano systems.

The outline of this paper is as follows: We will introduce the meshfree particle methods; The Cauchy-Born rule will then be implemented into the meshfree particle methods, which can also be coupled with molecular dynamics; Several examples are studied in the following section and the last section presents the conclusions.

\section{Meshfree Particle Methods at the Nanoscale}

\subsection{Discrete Equations}

The physical princ iples governing the continuum are the conservation of mass, momentum and energy. A so-called total Lagrangian description is employed (see Belytschko, Liu and Moran [12]); therefore, the linear momentum equations are

$$
\frac{\partial P_{j i}}{\partial X_{j}}+\rho_{0} b_{i}=\rho_{0} \ddot{u}_{i} .
$$

where $\rho_{0}$ is the initial density, $\mathbf{P}$ is the first Piola-Kirchhoff stress tensor, $\mathbf{X}$ is the reference coordinates, $\mathbf{b}$ is the body force per unit mass, $\mathbf{u}$ is the displacement and the superposed dots denote material time derivatives. The weak form of the momentum conservation equation is

$$
\int_{\Omega_{0}} \delta u_{i} \rho_{0} \ddot{u}_{i} d \Omega_{0}=\int_{\Omega_{0}} \delta u_{i} \rho_{0} b_{i} d \Omega_{0}-\int_{\Omega_{0}} \delta F_{i j} P_{j i} d \Omega_{0}+\int_{\Gamma_{0}} \delta u_{i} \bar{t}_{i} d \Gamma_{0}
$$

where $\Omega_{0}$ is the reference configuration, $\delta u_{i}$ is the test function, $F_{i j}$ is the gradient of deformation and $\bar{t}_{i}$ is the prescribed boundary traction. The particle approximation is 


$$
u_{i}(\mathbf{X}, t)=\sum_{I} w_{I}(\mathbf{X}) u_{i I}(t)
$$

where $w_{I}(\mathbf{X})$ is a Lagrangian kernel function, which is the function of reference coordinates. With a similar expansion for $\delta \mathbf{u}(\mathbf{X})$, the following discrete equations can be obtained:

$$
M_{I} \ddot{u}_{i I}=F_{i I}^{e x t}-F_{i I}^{i n t}, \quad M_{I}=\rho_{0} V_{I}^{o} .
$$

where $V_{I}^{0}$ is the volume associated with particle $I$ in the reference configuration. $F_{i I}^{e x t}$ and $F_{i I}^{i n t}$ are the external and internal nodal forces, respectively, given by

$$
F_{i I}^{e x t}=\int_{\Omega_{0}} \rho_{0} w_{I} b_{i} d \Omega+\int_{\Gamma_{0}^{t}} N_{I} \bar{t}_{i} d \Gamma_{0}, \quad F_{i I}^{i n t}=\int_{\Omega_{0}} \frac{\partial w_{I}(\mathbf{X})}{\partial X_{j}} P_{j i} d \Omega_{0}
$$

If the nodal integration scheme [11] is used in the meshfree particle methods, the internal nodal forces in (5) can be calculated by

$$
F_{i I}^{i n t}=\sum_{J} V_{J}^{0} \frac{\partial w_{I}\left(\mathbf{X}_{\mathbf{J}}\right)}{\partial X_{j}} P_{j i}\left(\mathbf{X}_{\mathbf{J}}\right)
$$

The Nodal integration scheme may result in one of instabilities due to the rank deficiency. A stress point integration scheme [11] can be used to stabilize it.

\subsection{Implementation of the Quasicontinuum Technique}

In a continuum model, the potential energy depends on the elongations and angle changes of the bonds at the atomistic level. The total potential of the continuum model can be written as

$$
W^{C}=\int_{\Omega_{0}} w_{C} d \Omega
$$

where $w_{C}$ is the potential energy per unit volume. Then, the first Piola-Kirchhoff stress can be obtained from the potential of the continuum by

$$
\mathbf{P}=\frac{\partial w_{C}(\mathbf{F})}{\partial \mathbf{F}} .
$$

where $\mathbf{F}$ is the deformation gradient. In this paper, it is assumed the molecular structure in the volume associated with each particle is under a constant deformation gradient. Therefore, the first Piola-Kirchhoff stress at each particle can be evaluated through (8). In other words, (8) serves as the constitutive equation for meshfree particle methods at the nanoscale. For curved monolayer crystalline membranes such as nanotubes, an extension of the Cauchy-Born rule, called the exponential Cauchy-Born rule, can be used (see Arroyo and Belytschko [9]). 


\subsection{Coupling with Molecular Dynamics}

Belytschko and Xiao [7] proposed a multiscale method called the bridging domain coupling method, in which, the molecular model and the continuum model overlap at their junctions in a bridging domain.

In this paper, molecular dynamics and the meshfree particle method are coupled via the bridging domain coupling technique. The complete domain in the initial configuration is denoted by $\Omega_{0}$. The domain is subdivided into the subdomain treated by continuum mechanics, $\Omega_{0}^{C}$, and the one treated by molecular dynamics, $\Omega_{0}^{M}$. The intersection of these two subdomains is called the bridging domain denoted by $\Omega_{0}^{\text {int }}$ in the initial configuration. The bridging domain multiscale modeling of a molecular chain is shown in Figure 1.

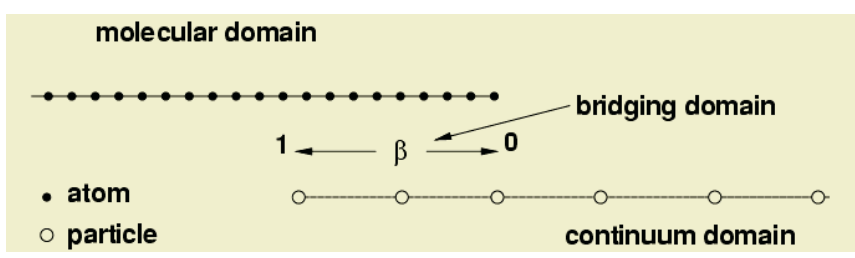

Fig. 1. A Bridging domain coupling model for a molecular chain

In expressing the total Hamiltonian of the system we employ a scaling parameter $\beta$ in the bridging domain as shown in Figure 1. The scaling parameter $\beta$ vanishes at one end of the bridging domain and is unity at another end. Therefore, the Hamiltonian for the complete domain is taken to be a linear combination of the molecular and continuum Hamiltonians

$$
\begin{aligned}
H=\beta H^{M}+(1-\beta) H^{C}= & \sum_{I} \beta\left(\mathbf{X}_{I}\right) \frac{\mathbf{p}_{I}^{M} \cdot \mathbf{p}_{I}^{M}}{2 m_{I}}+\beta W^{M} \\
& +\sum_{I}\left(1-\beta\left(\mathbf{X}_{I}\right)\right) \frac{\mathbf{p}_{I}^{C} \cdot \mathbf{p}_{I}^{C}}{2 M_{I}}+(1-\beta) W^{C}
\end{aligned} .
$$

where $W^{M}$ is the potential in the molecular model, and $W^{C}$ is the strain energy in the continuum model. The discrete equations can be obtained via the classical Hamiltonian mechanics. The details can be found in [7].

\section{Examples}

\subsection{Bending of a Nano Beam}

The bending of a nano cantilever beam is considered in this example. The nano beam contains 5,140 atoms and the dimensions are: length $L=270 \mathrm{~nm}$ and height $H=15.6 \mathrm{~nm}$. 
A pair potential function is used to approximate the interaction between nearest atoms,

$$
U(l)=0.5 k\left(l-l_{0}\right)^{2}
$$

where $k=10000 \mathrm{~N} / \mathrm{m}$ and $l_{0}=1 \mathrm{~nm}$.

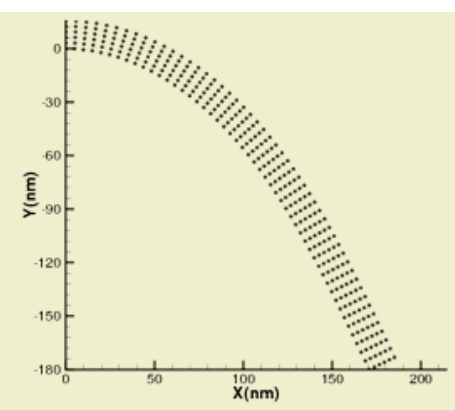

(a) The meshfree particle method

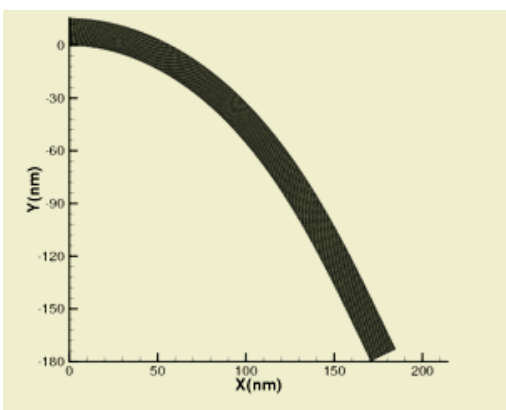

(b) The molecular mechanics calculation

Fig. 2. Deformed configurations of the nanobeam

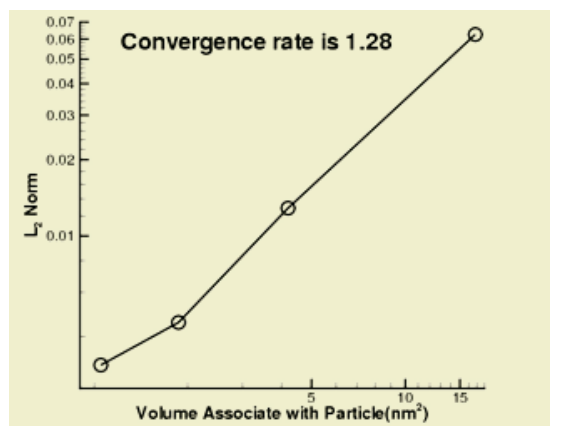

Fig. 3. Convergence of the nanoscale meshfree particle method

We use the meshfree particle method with 250 particles to simulate the bending of this nano beam. The prescribed displacement is applied on the right upper corner of the beam. The final configuration of the nano beam is shown in Figure 2(a). Figure 2(b) shows the deformed beam when performing the molecular mechanics calculation, and it supports the meshfree particle method result. As shown in figure 3, the convergence is also studied by using the $l_{2}$ error in displacement for the meshfree particle method.

\subsection{A Nano Plate with a Central Crack}

Meshfree particle methods are advantageous to simulate fracture problems. In this example, the meshfree particle method is used to study the stress concentration of a 


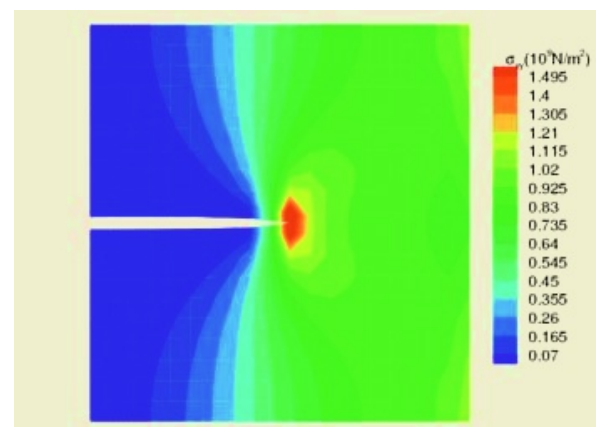

(a) The molecular mechanics calculation

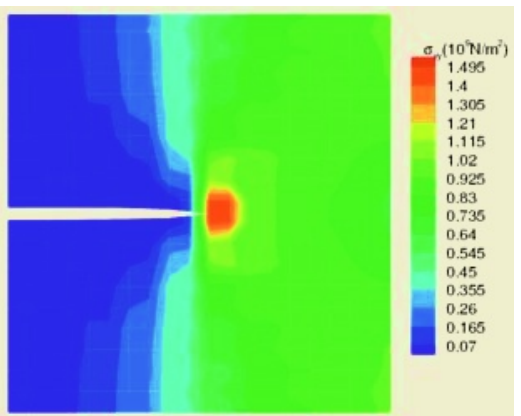

(b) The meshfree particle method

Fig. 4. Comparison of stress concentration at the crack tip

nano plate containing an central crack. This nano plate contains 86,915 atoms with the triangular molecular structure. The dimensions are: $L=270 \mathrm{~nm}$ and $M=280 \mathrm{~nm}$, and the crack length is $135 \mathrm{~nm}$. The crack is initialized by taking a number of bonds out. The meshfree particle model has 400 particles, and a visibility criterion is used to construct the kernel functions for the particles near the crack or around the crack tip. Figure 4 shows the comparison of the stress $\left(\sigma_{y y}\right)$ contour obtained from the molecular mechanics calculation with the one from the meshfree particle method. It can be seen that they are in accord.

\subsection{Wave Propagation in a Molecular Chain}

In this example, the wave propagation in a molecular chain, which contains 2001 atoms, is simulated. The LJ 6-12 potential function is used as the interatomic potential function between the nearest atoms, and it is

$$
w_{M}=4 \varepsilon\left[(\sigma / r)^{12}-(\sigma / r)^{6}\right] .
$$

where the constants are chosen as: $\sigma=3.4 e^{-10} \mathrm{~m}$ and $\varepsilon=1.65 e^{-21} \mathrm{~J}$. The mass of each atom is set to be $3.8 \times 10^{-10} \mathrm{~kg}$.

In the bridging domain coupling modeling of this molecule chain, there are 1001 atoms in the molecular domain and 200 particles in the continuum domain. The initial wave is the combination of high frequency and low frequency waves and starts to propagate from the molecular domain. A non-physical phenomenon, shown in Figure 5(a), can be observed if using a handshake coupling technique [4] without the application of the artificial viscosity. It is possible to see that the high frequency wave is reflected while the low frequency wave passes the continuum domain. Such a phenomenon is also called the spurious wave reflection. However, with the bridging domain coupling technique, the spurious wave reflection can be eliminated as shown in Figure 5(b). 


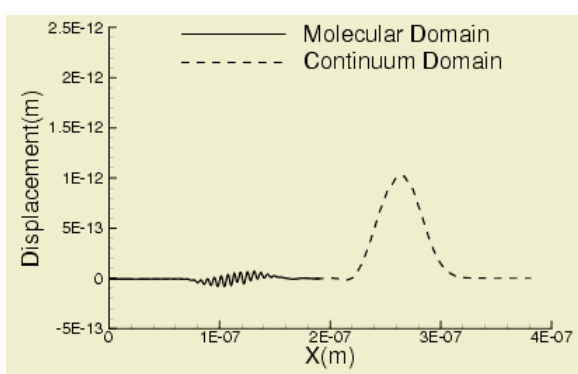

(a) The handshake method

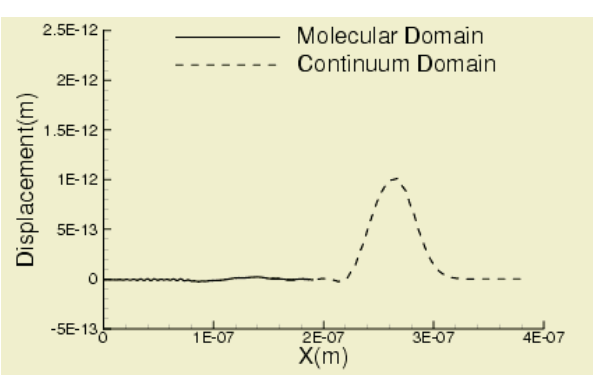

(b) The bridging domain coupling method

Fig. 5. Multiscale simulations on the wave propagation in a molecular chain

\section{Conclusions}

In this paper, the quasicontinuum technique (the Cauchy-Born rule) was implemented into the meshfree particle methods. Therefore, numerical simulations in nanotechnology can be valuable in regards to the meshfree particle methods. This progress makes it possible to treat extremely large deformation problems and the problems involving discontinuities, such as fractures, at the nanoscale. The examples showed that the nanoscale meshfree particle methods can give accurate results when compared with the molecular mechanics calculation outcomes. In addition, the meshfree particle methods can be coupled with molecular dynamics via the bridging domain coupling technique. The spurious wave reflection can be eliminated without any additional filtering processes.

\section{References}

1. Rountree, C. L., Kalia, R. K., Lidorikis, E., Nakano, A., Van, B. L., Vashishta, P.: Atomistic aspects of crack propagation in brittle materials: Multimillion atom molecular dynamics simulation. Annu. Rev. Mater. Res. 32(2002) 377-400

2. Abraham, F. F., Gao, H.: How fast can crack move. Phys. Rev. Lett. 84(2000) 3113-3116

3. Tadmor, E. B., Phillips, R., Ortiz, M.: Hierarchical modeling in the mechanics of materials. Int. J. Solids Struct. 37 (2000) 379-389

4. Abraham, F., Broughton, J., Bernstein, N., Kaxiras, E.: Spanning the continuum to quantum length scales in a dynamic simulation of brittle fracture. Europhys. Lett. 44(1998), 783-787

5. Rudd, R. E., Broughton, J. Q.: Coarse-grained molecular dynamics and the atomic limit of finite elements. Phys. Rev. B. 58(1998) R5893-R5896

6. Wagner, G. J., Liu, W. K.: Coupling of atomic and continuum simulations using a bridging scale decomposition. J. Comp. Phys. 190(2003) 249-274

7. Xiao, S., Belytschko, T.: A bridging domain method for coupling continua with molecular dynamics. Comput. Method Appl. M. (2004) in press

8. Tadmor, E. B., Ortiz, M., Phillips, R.: Quasicontinuum analysis of defects in solids. Philos. Mag. A 73(1996) 1529-1563 
9. Arroyo, M., Belytschko, T.: A finite deformation membrane based on inter-atomic potentials for the transverse mechanics of nanotubes. Mech. Mater. 35(2003) 193-215

10. Belytschko, T., Krongauz, K., Organ, D., Fleming, M., Krysl, P.: Meshless methods: An overview and recent developments. Comput. Method Appl. M. 139(1996) 3-47

11. Belytschko, T., Xiao, S.: Stability analysis of particle methods with corrected derivatives. Comput. Math. Appl. 43(2002) 329-350

12. Belytschko, T., Liu, W. K., Moran, B.: Nonlinear Finite Elements for Continua and Structures. Wiley, New York (2000) 